\title{
Compulsive Overeating as an Addictive Behavior: Overlap Between Food Addiction and Binge Eating Disorder
}

\author{
Caroline Davis
}

Published online: 13 February 2013

(C) Springer Science+Business Media New York 2013

\begin{abstract}
With the rise in worldwide rates of obesity in the past few decades, scientists and clinicians have focused considerable attention on trying to understand the causes of excessive and compulsive food intake - patterns of overeating that are highly correlated with body mass index (BMI). Given that prominent psycho-behavioral similarities exist between chronic binge eating and drug abuse, many have adopted the perspective that an apparent dependence on highly palatable food - accompanied by marked emotional and social distress and deficiency - is, in essence, an addiction disorder. This narrative review considers the overlapping symptoms and characteristics of binge eating disorder (BED), and models of food addiction, both in preclinical animal studies and in human research. It is concluded that overeating may best be viewed along a dimension reflecting degrees of severity and compulsiveness and that the high end of the continuum marks the clinically significant impairment seen in BED. We further suggest that what we have come to call 'food addiction' may simply be a more acute and pathologically-dense form of BED.
\end{abstract}

Keywords Binge eating disorder · Food addiction .

Dopamine $\cdot$ Obesity $\cdot$ Responsiveness to reward

\section{Introduction}

Obesity now ranks as one of the greatest and most preventable global threats to public health. It is also a highly transmissible condition ( .6 to .8$)$ suggesting a mainly genetic influence on population variation in Body Mass Index (BMI) [1, 2].

\section{Davis $(\bowtie)$}

Kinesiology \& Health Sciences, Faculty of Health,

York University, 343 Bethune College, 4700 Keele Street,

Toronto, ON, M3J1P3, Canada

e-mail: cdavis@yorku.ca
Heritability estimates are also greater in women than in men $[3,4]$. While the prevalence of obesity has doubled in the past few decades, its morbid form (BMI>40) has seen an alarming 4-fold increase [5]. In order to inform more personalized treatment approaches for those who struggle with overeating and weight gain, it behooves researchers to identify clinically relevant subtypes of obesity with specific psychobiological profiles that may convey different vulnerabilities to environmental risk factors, and therefore indicate different targets for intervention.

The parallels between an apparent 'loss-of-control' over food intake and compulsive drug taking have been recognized by clinicians for decades, albeit mostly in circumstantial reports, and with particular reference to the pronounced cravings they have in common and the similarities in their mood-enhancing effects [6-13]. Among the general public, the notion that overeating can be an addictive behavior has also been widely accepted for many years - as seen, for instance, by the numerous 12-Step treatment programs (e.g. Overeaters Anonymous founded in 1960; Food Addicts Anonymous founded in 1987) established for those who suffer with this problem.

In recent years - and notwithstanding the absence of a formal, or agreed-upon, set of diagnostic criteria - the notion of an addiction to hyper-palatable food is currently receiving widespread attention from the popular media. By contrast, it is only very recently that the term 'food addiction' has gained any scientific credibility. For instance, since 2008, according to the Web of Science data base, there has been a steeply exponential increase in the number of scientific publications devoted to this topic, with only two publications between 2000 and 2007, and 34 publications in 2012 alone. An important catalyst for the renewed interest in food addiction is the compelling and accumulating bio-behavioral evidence that highly palatable foods rich in sugar, fat, and salt have an abuse potential similar to addictive drugs like cocaine and alcohol [14-16]. In addition, evidence of the biological parallels between drug and food abuse, as demonstrated by 
preclinical experiments, human brain neuroimaging studies, and behavior genetic research, has further strengthened the credibility of the food-addiction construct.

\section{Animal Models of Food Addiction}

Rodent models of food addiction have typically used behavioral paradigms based on experimentally-inspired analogues of the Diagnostic and Statistical Manual (DSM-IV-TR) [17] criteria for substance dependence. For example, "tolerance" has been operationalized as an increase in brain-stimulation reward (BSR) thresholds following extended exposure to addictive drugs [18•]. Extrapolating this principle to eating behaviors, rats with 18-23 hours/day access to palatable energy-dense food displayed a worsening deficit in brain reward function, as seen by their progressively elevated BSR thresholds across days, compared to rats on a standard chow diet [18•].

Similarly, escalation of intake over time has been used as a marker of the criterion "taking the substance in larger amounts than was intended" [19]. In this regard, there is reliable evidence that rats fed an intermittent diet of sugar, develop a pattern of copious consumption resembling human cases of binge eating $[20,21 \bullet \cdot$. These studies show that a sugarenhanced diet increases daily food intake over time, and that following its removal, the animals show aggression, anxiety, teeth-chattering, and head-shaking - all symptoms associated with "withdrawal" from drugs like heroin. Similar increased intake has been found when animals were given high-fat [22] or other highly palatable diets [18•]. Evidence of withdrawal has also been observed in animals showing depressive-like symptoms after access to highly palatable food for 7 weeks, as indicated by increased immobility in the forced-swim test and decreased intake in the sucrose consumption test [23]. Interestingly, some of the depressive symptoms were abolished in these animals when they were given renewed access to the palatable diet, suggesting that compulsive eating can relieve withdrawalinduced negative affect, as well as fostering its onset.

It also appears that compulsive patterns of palatable food intake only need a short time to develop, and that rats rapidly display a lack of flexibility in their food choices by rejecting standard chow even when the greatly preferred rich 'cafeteria diet' is transiently unavailable [24]. Even very limited (10 $\mathrm{min}$ ) daily access to sugar-fat diets leads to binge-like intake and excessive weight gain in rats, but interestingly, without the stress and anxiety withdrawal responses seen in rats with access to sugar-rich diets [25] - a finding which adds to the growing evidence that only minimal withdrawal symptoms are associated with diets rich in fat, or a combination of sugar and fat, compared to the overt withdrawal symptoms following abstinence from a high-sugar diet [26, 27]. Various explanations have been proffered for these differences including the greater hedonic intensity of sugar relative to fat
[25], and the more pronounced opioid-activating potential of sugar [26]. In general, it appears that high fat consumption is largely responsible for weight gain while sugar may be liable for producing addictive-like behaviors including pronounced withdrawal symptoms [28].

Binge-prone female rats were also found to tolerate significantly higher levels of foot shock for access to Oreo cookies than their binge-resistant counterparts, confirming the "continued use despite negative consequences" criteria for substance dependence [29]. Other evidence of abnormal motivation for sweet and fatty foods was demonstrated when rats with extended exposure to a highly palatable cafeteria diet continued to consume this food even when it was laced with quinine to produce an unpleasant bitter taste [24]. Finally, while numerous studies have found behavioral and consummatory cross-sensitization from one addictive drug to another [21••] - and between drugs and stress [30] - there is now good evidence that sugar intake also crosssensitizes to drugs of abuse, and vice versa $[21 \bullet \bullet]$.

In conclusion, an important consideration in any model of addiction is the existence of great variability in the proneness to its development [31]. Only a subgroup of animals and people who are exposed to drugs of abuse, or highlypalatable food, seem to develop full-blown symptoms of dependence and loss-of-control over their intake $[32,33]-$ an observation that has focused considerable research attention on identifying the ingredients of risk, and the biological mechanisms underlying vulnerability [34]. For instance, there is some evidence that early-life stressors, and various dietary differences that arise from these stressors, are influential in the susceptibility to compulsive intake of palatable foods [31].

When evaluating animal paradigms that support the foodaddiction construct, another important consideration is that a pattern of compulsive intake - closely analogous to what we term binge eating in the human condition - is a universal feature of all models $[31,35 \bullet \cdot]$. In other words, animals do not display symptoms of withdrawal, tolerance, or any of the other analogue criteria for substance abuse, without also showing binge-like consumption of the experimental (high sweet/fat) food.

\section{Human Studies of Food Addiction}

Despite the robust evidence of sugar/fat dependence in rodents, there are few parallel findings in human research except for anecdotal reports and various qualitative clinical accounts [36-38, 39・•] Recently, researchers at Yale University moved the field forward with their development of a measure which operationalizes 'food addiction' using the DSM-IV-TR diagnostic criteria for substance dependence [40••]. Their preliminary evidence suggests that the Yale Food Addiction Scale (YFAS) has high convergent validity 
with other measures of eating pathology, especially binge eating, and may therefore be a useful tool for identifying individuals with addictive tendencies toward food.

In 2011, our group published the first case-control study using the YFAS diagnostic criteria in adult men and women $[40 \bullet \bullet$. The results provided very good evidence that food addiction is an identifiable clinical syndrome with psychiatric co-morbidities and a psycho-behavioral profile remarkably similar to conventional drug-abuse disorders. For example, those who met the diagnostic criteria for YFAS food addiction had a significantly greater co-morbidity with BED, severe depression, and attention-deficit/hyperactivity disorder (ADHD) compared to their age- and weight-equivalent counterparts. They were also more impulsive and displayed greater emotional reactivity than the obese controls. In addition, they reported more intense food cravings and were more likely to 'self-soothe' with food.

Based on experimental evidence that tryptophan depletion tends to foster high consumption of sweet/fatty foods, and that it can also trigger depressive episodes, some have argued that overeating of highly palatable foods is fundamentally a form of self-medication to ameliorate dysphoric mood [41•]. In light of the significantly more severe levels of depression in those with food addiction, and their reported proneness to emotionally-driven eating, these inter-relationships may well explain a downwardly spiraling cycle whereby a biological predisposition to negative mood (e.g., low 5-HT availability) could foster a voracious appetite for sugary and fatty foods. In our highly obesigenic environment with its proliferation of processed foods, this hungriness can easily be satisfied, in turn, down regulating brain reward pathways and further contributing to dysphoria and its consequences on eating behaviors.

To date, only one neuroimaging study has examined correlates of food addiction, and found that rewardcircuitry activation in the caudate and amygdala, in response to the anticipated receipt of food, was positively associated with YFAS-symptom scores [42]. Unfortunately, this study was somewhat limited by a small all-female sample, and because only two participants met the diagnostic criteria for food addiction. However, in a recent study - and the first to provide genetic support for the food-addiction construct our findings meshed well with these neuroimaging data [43]. A multilocus genetic index score, which reflected the cumulative effect of several functional markers on striatal dopamine signaling strength, was significantly higher in a group of overweight adults with YFAS food addiction compared to their age- and BMI-matched counterparts. This relationship was also mediated by greater food cravings, bingeing, and emotional eating. Together, these findings support the view that food addiction is a reward-responsive phenotype of obesity. They also suggest a reward-based process model whereby an inherent biological susceptibility contributes to increased risk for overeating, which in turn may promote addictive tendencies toward certain highly palatable foods.

\section{Binge Eating Disorder}

Binge eating disorder (BED) is an overeating syndrome seen much more frequently in those who are substantially overweight [44-46]. It also appears to be a behaviorally-distinct subtype of obesity with an unique risk profile reflecting a heightened response to rewarding stimuli. In particular, individuals with this disorder display a hyper-reactivity to the hedonic properties of food as seen by greater food cravings, preoccupation with thoughts of food, emotionally-induced overeating, and a greater preference for sweet and fatty foods $[47,48]$. While estimates suggest that the genetic contribution to $\mathrm{BED}$ is about $50 \%$, its prevalence is significantly greater in women than in men [49-51]. Moreover, it is no longer seen as a syndrome exclusive to adulthood. In recent years, bingeing or 'loss-of-control' eating has also become prevalent in middle childhood and early adolescence [52].

The research has consistently demonstrated that repeated consumption of highly palatable food can produce dopamine signaling changes in the brain, which promote an escalation of intake [53]. It also appears that intermittently restricted access to highly palatable foods results in sustained stimulation of the reward system, which also contributes to chronic overeating and to binge-eating susceptibility [54]. In this regard, it is relevant that individuals with BED tend to under-eat during the mornings [55], but show a propensity for high-calorie nocturnal eating [56], and do greater snacking in the evenings [57] compared to weightmatched adults who do not binge eat.

Neuro-scientific evidence of high reward sensitivity in BED can be found in the research showing greater medial orbitofrontal cortex reactivity to food cues in people with this disorder relative to comparison groups [58] - a finding which accords with the important role of this brain area in drug cravings, and which confirms the significance of dopaminergic mechanisms in the etiology of BED [59, 60]. Larger brain-activation differences in the amygdala and ventral striatum have also been found in obese adults with BED compared to weight-matched controls, suggesting a greater motivational sensitivity to, and visual processing of, palatable food cues in the former group [61]. The finding that obese binge eaters have greater increases in extracellular dopamine levels in the caudate nucleus (though not the ventral striatum) during food stimulation, compared to non-binge eating controls, provides further evidence of a hyper-responsiveness to reward in BED [62]. Additionally, a recent study has shown that exposure to personally-relevant, high-calorie food cues elicited hyper-responsive fMRI BOLD reactions in overweight adults with binge-eating behavior, and importantly, that the magnitude of these responses increased with greater number 
of binge-eating symptoms [63]. Finally, and again in response to food cues, binge eating women showed hyper-responsiveness and increased neural communication between the ventral striatum and a widespread network involved in reward and action planning compared to weight-matched controls [64].

In concurrence with the behavioral and neuroimaging evidence that BED reflects a heightened responsiveness to reward (described above), we recently reported that genetic markers reflecting enhanced dopaminergic responsiveness were positively related to the food-related sub-phenotypes of BED such as binge eating, food cravings, and emotional eating [65••]. In addition - and similar to findings in those with YFAS-diagnosed food addiction - a dopamine-based, multilocus genetic profile score was significantly higher in a group of obese adults with BED compared to their age- and BMI-matched counterparts [66].

\section{Overeating as a Spectrum of Compulsion and Severity}

As we have seen, the available research - albeit still very limited as it pertains to the human condition - consistently endorses the view that food addiction is an identifiable and viable clinical entity with many similarities to drug-addiction disorders. At the same time, however, these investigations, and related work, have implicitly raised the question of whether the YFAS-inspired definition of food addiction is in essence, and simply, a more severe expression of BED. This issue is especially germane given the substantial symptom overlap between the two conditions $[40 \bullet \bullet, 67 \bullet \bullet]$, the many co-morbidities they have in common [40••], and their similar biological underpinnings $[43,66]$. The studies by Davis et al. [40••] and Gearhardt et al. [67••] found that about $50 \%$ of obese adults diagnosed with YFAS food addiction also met criteria for BED. Moreover, in an earlier study [68], using structured telephone interviews in women diagnosed with BED, it was found that $92 \%$ of their sample met the DSM-IV-TR [17] criteria for substance dependence when the word "food" was substituted for "drug" in the interview questions. Clarifying whether the two conditions are sufficiently different in etiology and clinical course to warrant classification as separate pathological phenotypes of overeating is an important next-step in eating-disorder and addiction research. Resolution will also help guide personalized treatment strategies for those who struggle with intractable and excessive food consumption.

As an initial attempt to address this matter, we examined group differences on several variables related to demographic characteristics (age and BMI), patterns of overeating, personality risk factors, and co-morbid clinical symptoms, using independent t-test procedures. In the first set of analyses, we compared two equivalent groups of overweight men and women who met DSM criteria for BED: one with co-occurring YFAS-diagnosed food addiction $(n=16)$ and the other without $(n=17)$. Results indicated that the two BED groups were equivalent in age and BMI. On almost all other variables, however, the differences were highly statistically significant (see Table 1). Those with food addiction were much more likely to overeat for emotional and cue-driven reasons, they had more severe binge eating and food cravings, and they were more responsive to the rewarding properties of food. This group also had more addictive personality traits, was more impulsive, and had greatly elevated symptoms of depression compared to their non-food addict BED counterparts. While the food-addict group also reported greater ADHD symptoms, both currently and in childhood, these differences only approached statistical significance. Collectively, these findings mesh well with a related study of food addiction in BED patients. In particular, the number of YFAS symptoms (scores ranging from 0-7) was shown to correlate positively with Beck depression ratings, poor self-esteem, and difficulties in emotion regulation, as well as with certain measures of eating pathology including frequency of binge episodes [67••].

In our second analysis, we compared the BED group without food addiction to a weight- and age-matched group of adults without BED or food addiction $(n=66)$ on the same set of variables. By contrast to the first series of comparisons, these two groups were remarkably similar to each other, with only very few significant differences. Specifically, and not surprisingly, the BED group had higher binge-eating scores than the non-BED controls. They also reported more hedonic eating and elevated food cravings (see Table 1).

Despite the novel and interesting findings from this preliminary investigation, it is important to acknowledge that the results are based on a relatively small sample of participants with BED. Nevertheless, the highly statistically significant findings from the first set of analyses indicate that the sample was not under-powered since the mean differences were sufficiently large, relative to their standard errors, to demonstrate strong effect sizes. Unfortunately, however, in the total sample of mainly overweight adults, there were only six individuals without a diagnosis either of BED or of food addiction, precluding a full factorial two-way analysis of the data. Given the established associations between psychological/behavioral pathology and BMI in binge-eating related disorders, such a skewed diagnostic distribution is, unfortunately, liable to be a problem for all future comparative research in this area.

While normal-weight adults comprise only a small minority of those with BED, they appear to be another behaviorally-distinct subset of the BED population, suggesting that body weight is also an important feature or correlate of the overeating spectrum. For example, some studies have found that normal-weight BED adults are younger [69, 70], and that their binge episodes are less frequent [70] and of shorter duration than their overweight/obese counterparts [69]. Especially relevant are the findings that normal-weight $\mathrm{BED}$ is characterized by a greater 'drive for thinness' [71], 
Table 1 Means, standard deviations, and p-value differences between those with YFAS-diagnosed food addiction $[\mathrm{FA}](\mathrm{n}=16)$ and those without $[$ Non-FA] $(n=17)$ in a sample of adults with binge eating disorder (BED), and between Non-FA BED and obese adults without BED or YFAS-FA $(n=66)$

\begin{tabular}{|c|c|c|c|c|c|c|c|c|}
\hline \multirow[t]{3}{*}{ Variable } & \multicolumn{5}{|l|}{ BED } & \multirow{2}{*}{\multicolumn{3}{|c|}{$\frac{\text { OBESE }}{\text { Non-BED/FA }}$}} \\
\hline & \multicolumn{2}{|l|}{ FA } & \multicolumn{3}{|c|}{ Non-FA } & & & \\
\hline & Mean & SD & Mean & SD & $p=$ & Mean & SD & $p=$ \\
\hline Age & 34.2 & 5.6 & 31.8 & 6.1 & 0.254 & 32.6 & & 0.649 \\
\hline BMI & 37.1 & 5.6 & 39.2 & 5.1 & 0.258 & 37.5 & 6.2 & 0.291 \\
\hline Emotional eating [80] & 4.1 & 0.5 & 3.2 & 0.9 & 0.002 & 3.0 & 0.9 & 0.343 \\
\hline External eating [80] & 3.8 & 0.4 & 3.4 & 0.6 & 0.034 & 3.4 & 0.6 & 0.939 \\
\hline Binge eating [81] & 4.5 & 0.7 & 3.2 & 1.2 & 0.001 & 1.8 & 1.5 & $<0.0001$ \\
\hline Hedonic eating [82] & 82.9 & 15.4 & 64.1 & 18.0 & 0.003 & 53.0 & 17.5 & 0.023 \\
\hline Food cravings [83] & 177.0 & 25.2 & 139.3 & 37.0 & 0.002 & 120.4 & 31.8 & 0.038 \\
\hline Addictive personality traits [84] & 17.9 & 5.3 & 12.5 & 4.2 & 0.003 & 12.3 & 4.2 & 0.868 \\
\hline Impulsivity [85] & 71.3 & 11.5 & 60.0 & 7.9 & 0.002 & 64.6 & 11.2 & 0.109 \\
\hline Depression symptoms [86] & 21.2 & 13.8 & 10.4 & 7.4 & 0.008 & 10.6 & 8.5 & 0.925 \\
\hline ADHD symptoms (adult) [87] & 35.4 & 17.2 & 25.6 & 13.5 & 0.076 & 25.2 & 12.7 & 0.908 \\
\hline ADHD symptoms (child) [88] & 37.6 & 24.0 & 24.2 & 17.0 & 0.073 & 25.5 & 18.3 & 0.798 \\
\hline
\end{tabular}

more pronounced cognitive and dietary restraint [72], and significantly greater and more extreme compensatory weight-control behaviors like skipping meals, exercising assiduously, and restricting intake [69].

Not surprisingly, a diagnosis of BED has been shown to predict weight gain over time [73, 74]. Indeed it is physiologically axiomatic that chronic episodes of binge eating in the absence of any compensation for the excessive ingestion of energy put individuals at substantial risk for obesity as they age. Therefore - and because one of the diagnostic criteria for BED is the absence of "inappropriate compensatory behaviors like purging, fasting, and excessive exercise" - the boundary between normal-weight BED and non-purging bulimia nervosa is clearly blurred at best, and their differences appear to be largely a matter of calorie-sparing severity.

Considering the full complement of research, including the pattern of response across our three study groups, findings strongly suggest that overeating can be viewed as a dimension reflecting its severity and its degree of compulsiveness (see Fig. 1). At the low end is passive overeating with little to no psychopathology, then mild and intermittent binge eating, followed by sub-groups of BED with some scarcely indistinguishable from normal-weight adults with nonpurging bulimia nervosa. Among overweight adults with BED — a group who comprise the large majority of those with BED—some manifest virtually no psychopathological or behaviorally-aberrant differences from BMI-matched adults without BED except for their binge eating, and their elevated responsiveness to highly palatable food. Others, however specifically those who meet the proposed YFAS criteria for food addiction - express significantly more severe and compulsive symptomatology, and psychopathological characteristics akin to individuals with conventional drug-addiction disorders. In other words, at the low end of the continuum are those who - despite excessive calorie intake and weight gain - display little or no behavioral pathology and psychiatric disturbances, followed by those with a similar lack of pathology except in relation to their compulsive binge eating. At the high end of the continuum are those with BED who display significant psychopathology and strong addictive tendencies toward food. In particular, they are highly impulsive, manifest significant mood disturbances, and display emotionallydriven bouts of overeating.

\section{Concluding Remarks}

As we have seen in this review, many subscribe to the view that compulsive overeating - a quintessentially-defining feature of BED - shares pronounced similarities with conventional addictions like substance abuse [31, 75•, 76, 77]. Not surprisingly, such discussions have inspired cautious

\section{Normal Passive Overeating Bingeing BED 'Food Addiction'
Weight}

Fig. 1 Patterns of overeating: a spectrum of compulsion and severity 
conclusions that some forms of excessive overeating are most appropriately viewed as an addiction disorder. While evidence for the validity of the food-addiction construct is rapidly gaining ground, inevitably this perspective has also found its detractors [78]. Unfortunately, some participating on the negative side of the debate have, in presenting their arguments, conflated obesity and overeating with 'food addiction', which has clouded its distinctiveness and muddled efforts to properly assess its diagnostic utility.

It is clearly a misinterpretation of the research for anyone to perceive claims that all cases of overeating - even prodigious overeating — reflect an 'addiction to food', in the same way as it would be nonsensical to say that all heavy drinkers are alcoholics. In other words, there are no explicit assertions from advocates of the food-addiction concept that more than a subset of the population could, or would, be affected by this condition. Like virtually all psychiatric disorders, including drug abuse and behavioral addictions such as pathological gambling, there is a relatively low base rate in the population, even though the related symptoms and endo-phenotypes exist in the general population along a continuum of varying magnitude or severity. Most individuals who use addictive substances - drugs or highly palatable food - do not develop dependence. Many contributors explain an individual's propensity to these disorders. Moreover, it is highly unlikely that a homogeneous set of risk factors pertains to all phases of their development [79].

Acknowledgment C. Davis has received research support from the Canadian Institute of Health Research.

Disclosure No potential conflicts of interest relevant to this article were reported.

\section{References}

Papers of particular interest, published recently, have been highlighted as:

- Of importance,

•- Of major importance

1. Choquet H, Meyre D. Molecular basis of obesity: current status and future prospects. Curr Genomics. 2011;12:154-68.

2. Nan C, Guo B, Warner C, et al. Heritability of body mass index in pre-adolescence, young adulthood and late adulthood. Eur J Epidemiol. 2012;27:247-53.

3. Hur Y-M. Sex differences in heritability of BMI in South Korean adolescent twins. Obesity. 2007;15:2908-11.

4. Schousboe K, Willemsen G, Kyvik KO, et al. Sex differences in heritability of BMI: a comparative study of results from twin studies in eight countries. Twin Res. 2003;6:409-21.

5. Sturm R. Increases in clinically severe obesity in the United States, 1986-2000. Arch Internal Med. 2003;163:2146-8.

6. Davis C, Claridge G. The eating disorders as addiction: a psychobiological perspective. Addict Behav. 1998;23:463475.
7. Kayloe JC. Food addiction. Psychother. 1993;30:269-75.

8. McDowell M. Compulsive eating as a non-neurotic food addiction phenomenon. Obes Bariatric Med. 1978;7:140.

9. Randolph TG. Food allergy and food addiction. JAMA. 1953;153:821.

10. Randolph TG. The descriptive features of food addiction-addictive eating and drinking. Q J Stud Alcohol. 1956;17:198-224.

11. Randolph TG. Food addiction (chronic food allergy) - apparent basis of other addictions. Clin Res. 1978;26:A700.

12. Rogers PJ, Smit HJ. Craving and food addiction: a critical review of the evidence from a biopsychosocial perspective. Pharmacol Biochem Behav. 2000;66:3-14.

13. Tuomisto T, Hetherington MM, Morris MF, et al. Psychological and physiological characteristics of sweet food "addiction". Int $\mathrm{J}$ Eat Disord. 1999;25:169-75.

14. Gearhardt AN, Davis C, Kuschner R, et al. The addiction potential of hyperpalatable foods. Curr Drug Abuse Rev. 2011;4:140-5.

15. Kenny PJ. Reward mechanisms in obesity: New insights and future directions. Neuron. 2011;69:664-79.

16. Spring B, Schneider K, Smith M, et al. Abuse potential of carbohydrates for overweight carbohydrate cravers. Psychopharmacol. 2008;197:637-47.

17. American Psychiatric Association. Diagnostic and Statistical Manual - Version IV-TR. Washington. DC: American Psychiatric Association Press; 2000.

18. - Johnson PM, Kenny PJ. Dopamine D2 receptors in addiction-like reward dysfunction and compulsive eating in obese rats. Nat Neurosci. 2010;13:635-41. Changes in reward homeostasis in response to highly addictive drugs like cocaine and heroin have been observed in preclinical research. This paper presents evidence that the emergence of progressively worsening deficits in neural reward responses is also associated with the development of obesity.

19. Corwin RL, Grigson PS. Symposium overview — food addiction: fact or fiction? J Nutr. 2009;139:617-9.

20. Avena NM, Hoebel BG. A diet promoting sugar dependency causes behavioral cross-sensitization to a low dose of amphetamine. Neurosci. 2003;122:17-20.

21. •- Avena NM, Rada P, Hoebel BG. Evidence of sugar addiction: Behavioral and neurochemical effects of intermittent, excessive sugar intake. Neurosci Biobehav Rev. 2008;32:20-39. This comprehensive review summarizes evidence of sugar dependence in an animal model focusing on 4 components of conventional addiction disorders: bingeing, withdrawal, craving, and cross-sensitization. The evidence indicates that under certain conditions rats can become physiologically dependent to sugar.

22. Lutter M, Nestler EJ. Homeostatic and hedonic signals interact in the regulation of food intake. J Nutr. 2009;139:629-32.

23. Iemolo A, Valenza M, Tozier L, et al. Withdrawal from chronic, intermittent access to a highly palatable food induces depressivelike behavior in compulsive eating rats. Behav Pharmacol. 2012;23:593-602.

24. Heyne A, Kiesselbach C, Sahun I, et al. An animal model of compulsive food-taking behaviour. Addict Biol. 2009;14:373-83.

25. Parylak SL, Cottone $P$, Sabino V, et al. Effect of $\mathrm{CB}_{1}$ and $\mathrm{CRF}_{1}$ receptor antagonists on binge-like eating in rats with limited access to sweet fat diet: Lack of withdrawal-like responses. Physiol Behav. 2012;107:231-42.

26. Avena NM, Rada P, Hoebel BG. Sugar and fat bingeing have notable differences in addictive-like behaviors. J Nutr. 2009;139:623-8.

27. Bocarsly ME, Berner LA, Hoebel BG, et al. Rats that binge fat-rich food do not show somatic signs or anxiety associated with opiatelike withdrawal: Implications for nutrient-specific food addiction. Physiol Behav. 2011;104:865-72.

28. Avena NM. The study of food addiction using animal models of binge eating. Appetite. 2010;55:734-7. 
29. Oswald KD, Murdaugh DL, King VL, et al. Motivation for palatable food despite consequences in an animal model of binge eating. Int $\mathbf{J}$ Eating Disord. 2011;44:203-11.

30. Covington HE, Miczek KA. Repeated social defeat stress, cocaine or morphine. Effects on behavioral sensitization and intravenous cocaine self-administration "binges". Psychopharmacol (Berl). 2001;158:388-98.

31. Corwin RL, Avena NM, Boggiano MM. Feeding and reward: perspectives from three rat models of binge eating. Physiol Behav. 2011;104:87-97.

32. Deroche-Gamonet V, Berlin D, Piazza PV. Evidence for addictionlike behavior in the rat. Science. 2004;305:1014-7.

33. Reboussin BA, Anthony JC. Is there epidemiological evidence to support the ideas that a cocaine dependence syndrome emerges soon after onset of cocaine use? Neuropsychopharmacol. 2006;31:2055-64.

34. Boggiano MM, Artiga AI, Pritchett CE, et al. High intake of palatable food predicts binge-eating characteristics independent of susceptibility to obesity: An animal model of lean vs obesity binge eating and obesity with and without binge-eating. Int J Obes. 2007;31:1357-67.

35. • de Jong JW, Vanderschurne LJMJ, Adan RAH. Towards an animal model of food addiction. Obes Facts. 2012;5:180-95. Similarities between Binge Eating Disorder and drug addiction are reviewed. An animal model for addiction-and in this case, food addiction - focuses on high motivation to obtain the substance, persistence in this behavior, and continuation of drug taking despite negative consequences.

36. Ifland JR, Preuss HG, Marcus MT, et al. Refined food addiction: a classic substance use disorder. Med Hypotheses. 2009;72:518-26.

37. Ronel N, Libman G. Eating disorders and recovery: lessons from overeaters anonymous. Clin Soc Work J. 2003;31:155-71.

38. Russell-Mayhew S, von Ranson KM, Masson PC. How does overeaters anonymous help its members? A qualitative analysis. Eur Eat Disord Rev. 2010;18:33-42.

39. • Gearhardt AN, Corbin WR, Brownell KD. Preliminary validation of the Yale food addiction scale. Appetite. 2009;52:430-6. These authors report the development of the self-report inventory to assess addictive tendencies toward food, which is based strictly on the $D S M-I V-R$ criteria for substance dependence. This tool has been very important in moving forward human research on food addiction.

40. •- Davis C, Curtis C, Levitan RD, et al. Evidence that 'food addiction' is a valid phenotype of obesity. Appetite. 2011;57:711-7. This study is the first human case-control study of food addiction and demonstrated significant clinical, psychological, and co-morbid difference between obese adults diagnoses with food addiction according to the Yale Food Addiction scale and their non-food addicted counterparts. The former had a profile very close to what is seen in those with substance abuse/dependence.

41. - Fortuna JL. The obesity epidemic and food addiction: clinical similarities to drug dependence. J Psychoact Drugs. 2012;44 (1):56-63. There are several biological similarities between dependence of palatable food and drug dependence including cravings and loss of control. This paper reviews the evidence in the area and also points to several environmental factors that have contributed to the obesity epidemic.

42. Gearhardt AN, Yokum S, Orr PT, et al. Neural correlates of food addiction. Arch Gen Psychiatry. 2011;32:E1-9.

43. Davis C, Levitan RD, Kaplan AS, et al. Genetic evidence that 'food addiction' reflects enhanced dopamine responsiveness in brain reward pathways. Presented at the annual meeting of the Society for the Study of Ingestive Behaviors. Zurich, Switzerland; July 10-14, 2012.

44. Franko DL, Thompson-Brenner H, Thompson DR, et al. Racial/ ethnic differences in adults in randomized clinical trials of binge eating disorder. J Consult Clin Psychol. 2012;80:186-95.
45. Tanofsky-Kraff M, Shomaker LB, Stern EA, et al. Children's binge eating and development of metabolic syndrome. Int $\mathrm{J}$ Obes. 2012;36:956-62.

46. Grilo CM, White MA, Masheb RM. The metabolic syndrome and behavioral correlates in obese adults with binge eating disorder. Int J Eat Disord. 2009;42:228-34.

47. Davis C, Levitan RD, Carter JC, et al. Personality and eating behaviours: a case-control study of binge eating disorder. Int $\mathrm{J}$ Eat Disord. 2008;41:243-50.

48. Davis C, Levitan RD, Carter JC, et al. Dopamine for 'wanting' and opioids for 'liking': a comparison of overweight/obese adults with and without binge eating disorder. Obes. 2009;17:1220-5.

49. Bulik CM, Sullivan PF, Kendler KS. Genetic and environmental contributions to obesity and binge eating. Int $\mathrm{J}$ Eat Disord. 2003;33:293-8.

50. Javaras KN, Laird NM, Reichborn-Kjennerud T, et al. Familiality and heritability of binge eating disorder: Results of a case-control family study and a twin study. Int J Eat Disord. 2008;41:174-9.

51. Mitchell KS, Neale MC, Bulik CM, et al. Binge eating disorder: a symptom-level investigation of genetic and environmental influences on liability. Psychol Med. 2010;40:1899-906.

52. Tanofsky-Kraff M. Psychosocial preventive interventions for obesity and eating disorders in youth. Int Rev Psychiatry. 2012;24:262-70.

53. Bello NT, Hajnal A. Dopamine and binge eating behaviors. Pharmacol Biochem Behav. 2010;97:25-33.

54. Hajnal A, Nyland JE, Anderzhanova E, et al. Chronic intermittent access to highly palatable diets result in sustained and augmented accumbens dopamine release and abolished response to regular chow compared to continuous availability. Washington, DC: Society for Neuroscience; 2008.

55. Raymond NC, Neumeyer B, Warren CS, et al. Energy intake patterns in obese women with binge eating disorder. Obes Res. 2003;11:869-79.

56. Masheb RM, Grilo CM, White MA. An examination of eating patterns in community women with bulimia nervosa and binge eating disorder. Int J Eating Disord. 2011;44:618-24.

57. Harvey K, Rosselli F, Wilson GT, et al. Eating patterns in patients with spectrum binge-eating disorder. Int J Eat Disord. 2011;44:447-51.

58. Schienle A, Schafer A, Hermann A, et al. Binge eating disorder: reward sensitivity and brain activation to images of reward. Biol Psychiatry. 2009;65:654-61.

59. Franklin T, Wang Z, Suh JJ, et al. Effects of Varenicline on smoking cue-triggered neural and craving responses. Arch Gen Psychiatry. 2011;68:516-26.

60. Rolls ET. McCabe C. Enhanced affective brain representations of chocolate in cravers vs non-cravers Eur J Neurosci. 2007;26:1067-76.

61. Weygandt M, Schaefer A, Schienle A, et al. Diagnosing different binge-eating disorders based on reward-related brain activation patterns. Hum Brain Mapping. 2012;33:2135-46.

62. Wang G-J, Geliebter A, Volkow ND, et al. Enhanced striatal dopamine release during food stimulation in binge eating disorder. Obes (Silver Spring). 2012;19:1601-8.

63. Filbey FM, Myers US, DeWitt S. Reward circuit function in high BMI individuals with compulsive overeating: Similarities with addiction. Neuroimage. 2012;63:1800-6.

64. Benson L, Carnell S, Pantazatos S, et al. Binge eaters show greater brain activation and connectivity within appetitive network in response to food cues, independent of weight status. Obes (Silver Spring). 2011;19:(suppl1)-S192.

65. • Davis C, Levitan RD, Yilmaz Z, et al. Binge eating disorder and the dopamine D2 receptor: Genotypes and sub-phenotypes. Prog Neuropsychopharmacol Bio Psychiatry. 2012;38:328-35. Certain functional markers of genes regulating dopamine signaling in the brain striatal area were associated with binge eating disorder (BED) and eating-related sub-phenotypes of BED. Results support 
the view that BED may have its causal origins in a hyperresponsiveness to rewarding stimuli such as highly palatable food.

66. Davis C, Levitan RD, Carter JC, et al. Binge eating disorder and 'food addiction': A multi-locus genetic-profile study. Paper presented at the annual conference of the Eating Disorder Research Society. 2012, Porto, Portugal.

67. •- Gearhardt AN, White MA, Masheb RM, et al. An examination of the food addiction construct in obese patients with binge eating disorder. Int J Eat Disord. 2012;45:657-63. Those obese patients with Binge Eating Disorder who met the Yale Food Addiction Scale criteria for food addiction appeared to represent a more disturbed variation of this condition. They displayed greater eating-behaviour pathology and associated psychopathology.

68. Cassin SE, von Ranson KM. Is binge eating experienced as an addiction? Appetite. 2007;49:687-90.

69. Goldschmidt AB, Le Grange D, Powers P, et al. Eating disorder symptomatology in normal-weight vs. obese individuals with binge eating disorder. Obesity (Silver Spring). 2011;19:1515-8.

70. Dingemans AE, van Furth EF. Binge eating disorder psychopathology in normal weight and obese individuals. Int $\mathrm{J}$ Eat Disord. 2012;45:135-8.

71. Barry DT, Grilo CM, Masheb RM. Comparison of patients with bulimia nervosa, obese patients with binge eating disorder, and non-obese patients with binge eating disorder. J Nerv Ment Dis. 2003;191:589-4.

72. Carrad I, Van der Linden M, Golay A. Comparison of obese and nonobese individuals with binge eating disorder: delicate boundry between binge eating disorder and non-purging bulimia nervosa. Eur Eat Disorders Rev. 2012;20:350-4.

73. Fairburn CG, Cooper Z, Doll HA, et al. The natural course of bulimia nervosa and binge eating disorder in young women. Arch Gen Psychiatry. 2000;57:659-65.

74. Field AE, Sonneville KR, Micali N, et al. Prospective association of common eating disorders and adverse outcomes. Pediatrics. 2012;130:E289-95.
75. - Davis C, Carter JC. Compulsive overeating as an addiction disorder: a review of theory and evidence. Appetite. 2009;53:18. Compulsive overeating as an addiction disorder: A review of theory and evidence.

76. Gearhardt AN, Corbin WR. The role of food addiction in clinical research. Curr Pharmaceut Design. 2011;17:1140-2.

77. Meule A. How prevalent is "food addiction"? Frontiers in Psychiatry. 2011;2:1-4

78. Ziaudden H, Farooqi S, Fletcher PC. Obesity and the brain: how convincing is the addiction model? Nature Rev Neurosci. 2012;13:279-86.

79. Swendsen J, Le Moal M. Individual vulnerability to addiction. Ann NY Acad Sciences. 2011;1216:73-85.

80. Van Strien T, Frijters JE, Bergers GP, et al. The Dutch eating behavior questionnaire (DEBQ) for assessment of restrained, emotional, and external eating behavior. Int J Eating Disorders. 1986;5:295-315.

81. Halmi KA, Falk JR, Schwartz E. Binge eating and vomiting: a survey of a college population. Psychol Med. 1981;11:697-706.

82. Lowe MR, Butryn ML, Didie ER, et al. The power of food scale: a new measure of the psychological influence of the food environment. Appetite. 2009;53:114-8.

83. Cepeda-Benito A, Gleaves DH, Williams TL, et al. The development and validation of the state and trait food-cravings questionnaires. Behav Ther. 2000;31:151-73.

84. Eysenck HJ, Eysenck SBG. Manual of the eysenck personality questionnaire. London: Hodder \& Stoughton; 1975.

85. Patton JH, Stanford MS, Barratt ES. Factor structure of the Barratt impulsivity scale. J Clin Psychol. 1995;51:768-74.

86. Beck AT, Steer RA, Brown GK. Manual for the beck depression inventory (BDI-II). 2nd ed. San Antonio, TX: The Psychological Association; 1996.

87. Conners CK, Erhardt D, Sparrow E. Conners adult ADHD rating scales. New York: Multi-Health Systems Inc.; 1999.

88. Ward MF, Wender PH, Reimherr FW. The wender Utah rating scale: an aid in the retrospective diagnosis of childhood attention deficit hyperactivity disorder. Am J Psychiatry. 1993;150:885-90. 